\title{
Is conflict adaptation an illusion?
}

\author{
James R. Schmidt ${ }^{1 *}$, Wim Notebaert ${ }^{2}$ and Eva Van Den Bussche ${ }^{3}$ \\ ${ }^{1}$ Department of Experimental Clinical and Health Psychology, Ghent University, Ghent, Belgium \\ 2 Department of Experimental Psychology, Ghent University, Ghent, Belgium \\ ${ }^{3}$ Department of Psychology, Vrije Universiteit Brussel, Brussels, Belgium \\ *Correspondence: james.schmidt@ugent.be
}

Edited and reviewed by:

Bernhard Hommel, Leiden University, Netherlands

Keywords: conflict adaptation, contingency learning, cognitive control, attention, timing, expectancies, proportion congruent, congruency sequence effect

Conflict adaptation theory is one of the most popular theories in cognitive psychology. The theory argues that participants strategically modulate attention away from distracting stimulus features in response to conflict. This idea was particularly popularized with the publication of the conflict monitoring model of Botvinick et al. (2001). Although the conflict adaptation view is able to explain a wide range of results with a seemingly intuitive set of mechanisms, some researchers have expressed skepticism. The paradigms used in the study of conflict adaptation typically require the manipulation of stimulus frequencies, sequential dependencies, time-on-task regularities, and various other task regularities that introduce the potential for learning of conflictunrelated information (for a review, see Schmidt, 2013a). This raises the possibility that although the data patterns (e.g., reduced congruency effects following incongruent trials) might be very real, the conflict adaptation mechanism typically used to explain them might be an illusion.

This research topic produced 17 articles from 39 authors. The contributions span a range of tasks, broadly divided into work on the congruency sequence effect (CSE) and various versions of the proportion congruency (PC) task. Duthoo et al. (2014) provide an updated review of the CSE literature, including considerations regarding difficulties with learning confounds that will need to be overcome in future research. Braem et al. (2014) provide a review and synthesis of work on cross-task CSEs, and they highlight a potentially important role of similarity in task context. Egner (2014) provides another review wherein it is argued that "learning biases" and conflict adaptation may be two expressions of a similar learning mechanism, the latter merely more abstract than the former.

The role that feature bindings play in confounding the CSE has been a central issue since seminal papers by Mayr et al. (2003) and Hommel et al. (2004). Spapé and Hommel (2014) further this work with a paradigm in which target location boxes rotate to new positions on the screen between trials, with results seeming to indicate a dependency of CSEs on bindings between stimuli. Van Lierde et al. (2014) present masked-priming experiments that produced an irregular CSE pattern when feature repetitions were included, but a regular CSE in the error rates with feature repetitions excluded. Wendt et al. (2014) present data to suggest that controls for feature bindings may be insufficient in cross-task CSEs when there is a semantic overlap between features in the two sub-tasks.
As early as the very first observation of a CSE, the role of expectancies about a repetition vs. alternation of congruency type (i.e., congruent vs. incongruent) has been discussed (Gratton et al., 1992). Jiménez and Méndez (2014) present evidence to suggest that conscious expectancies only influence behavior when participants are explicitly probed for their expectancies. In a less traditional paradigm using alphabet verification and serial reaction tasks, Gaschler et al. (2014) present evidence for the transfer of control demands from one learning task to another.

Some key articles have illustrated the major issues with contingent regularities in PC and CSE tasks (e.g., Schmidt and Besner, 2008; Schmidt and De Houwer, 2011; Mordkoff, 2012). Hazeltine and Mordkoff (2014) observe that robust effects of contingencies fully account for item-specific PC (ISPC) effects (see also, Schmidt, 2013b). They further observe sequential modulations of both contingencies and congruency on the CSE. In contrast, Blais et al. (2014) suggest that contingency biases and "congruency switch" biases are unlikely to contribute to the CSE, though Schmidt (2014b) contests the interpretation of the data in a response paper.

A particularly interesting, howbeit controversial, development in the PC literature came with the suggestion that adaptation to conflict might occur in an item-specific (Jacoby et al., 2003) or context-specific fashion (Corballis and Gratton, 2003; Crump et al., 2006). Schmidt et al. (2014) present a non-conflict analog to the context-specific PC effect and argue that the "contextspecific proportion easy" effect they observe is consistent with the notion that context-specific rhythms might explain contextspecific PC effects. Atalay and Misirlisoy (2014) investigate the ISPC effect with different asynchronies (SOA) between targets and distracters. Generally consistent with a contingency learning perspective, they observe robust ISPC effects across lags, except when the distracting word came too late after the color.

Entel et al. (2014) investigate the influence of explicitly instructed contingencies on PC effects. They suggest that instructions alone might trigger proactive control, while also arguing an important role for contingencies. Hasegawa and Takahashi (2014) investigate block-wide PC effects and CSEs in a masked priming paradigm. They observed block-wide PC effects even with minimal stimulus awareness, but evidence for CSEs was limited to errors.

The topic closes with two opinion articles. Schmidt (2014a) discusses yet another potential caveat with contingency biases in 
cognitive control paradigms: if some stimuli are highly predictive of a response, whereas others are not, then differences in stimulus informativeness can lead to attentional capture biases. Finally, Levin and Tzelgov (2014) discuss an interesting distinction between task and informational conflict, and how this distinction might have important implications for theorizing in the cognitive control literature.

The range of perspectives presented in this research topic are as diverse as the questions assessed. Regarding the main question of interest (i.e., "Is Conflict Adaptation an Illusion?"), some authors argue that the answer is a resounding "yes," others argue that evidence for conflict adaptation is clear, and yet others fall somewhere in between. Whether or not conflict adaptation is merely an illusion is still an open question, but the contributions of the current research topic add interesting new layers to the debate. We hope that this research topic will open new avenues for research in the area that may lead to more definitive answers.

\section{ACKNOWLEDGMENTS}

JS (1211814N), WN (G.0098.09N), and EVDB (G023213N) are all supported by the Research Foundation-Flanders (FWOVlaanderen).

\section{REFERENCES}

Atalay, N. B., and Misirlisoy, M. (2014). ISPC effect is not observed when the word comes too late: a time course analysis. Front. Psychol. 5:1410. doi: 10.3389/fpsyg.2014.01410

Blais, C., Stefanidi, A., and Brewer, G. A. (2014). The Gratton effect remains after controlling for contingencies and stimulus repetitions. Front. Psychol. 5:1207. doi: 10.3389/fpsyg.2014.01207

Botvinick, M. M., Braver, T. S., Barch, D. M., Carter, C. S., and Cohen, J. D. (2001). Conflict monitoring and cognitive control. Psychol. Rev. 108, 624-652. doi: 10.1037/0033-295X.108.3.624

Braem, S., Abrahamse, E. L., Duthoo, W., and Notebaert, W. (2014). What determines the specificity of conflict adaptation? a review, critical analysis, and proposed synthesis. Front. Psychol. 5:1134. doi: 10.3389/fpsyg.2014.01134

Corballis, P. M., and Gratton, G. (2003). Independent control of processing strategies for different locations in the visual field. Biol. Psychol. 64, 191-209. doi: 10.1016/S0301-0511(03)00109-1

Crump, M. J., Gong, Z., and Milliken, B. (2006). The context-specific proportion congruent stroop effect: location as a contextual cue. Psychon. Bull. Rev. 13, 316-321. doi: 10.3758/BF03193850

Duthoo, W., Abrahamse, E. L., Braem, S., Boehler, C. N., and Notebaert, W. (2014). The heterogeneous world of congruency sequence effects: an update. Front. Psychol. 5:1001. doi: 10.3389/fpsyg.2014.01001

Egner, T. (2014). Creatures of habit (and control): a multi-level learning perspective on the modulation of congruency effects. Front. Psychol. 5:1247. doi: 10.3389/fpsyg.2014.01247

Entel, O., Tzelgov, J., and Bereby-Meyer, Y. (2014). Proportion congruency effects: instructions may be enough. Front. Psychol. 5:1108. doi: 10.3389/fpsyg.2014.01108

Gaschler, R., Marewski, J. N., Wenke, D., and Frensch, P. A. (2014). Transferring control demands across incidental learning tasks: stronger sequence usage in serial reaction task after shortcut option in letter string checking. Front. Psychol. 5:1388. doi: 10.3389/fpsyg.2014.01388

Gratton, G., Coles, M. G. H., and Donchin, E. (1992). Optimizing the use of information: strategic control of activation of responses. J. Exp. Psychol. 121, 480-506. doi: 10.1037/0096-3445.121.4.480

Hasegawa, K., and Takahashi, S. (2014). The role of visual awareness for conflict adaptation in the masked priming task: comparing block-wise adaptation with trial-by-trial adaptation. Front. Psychol. 5:1347. doi: 10.3389/fpsyg.2014. 01347
Hazeltine, E., and Mordkoff, J. T. (2014). Resolved but not forgotten: stroop conflict dredges up the past. Front. Psychol. 5:1327. doi: 10.3389/fpsyg.2014.01327

Hommel, B., Proctor, R. W., and Vu, K.-P. L. (2004). A feature-integration account of sequential effects in the Simon task. Psychol. Res. 68, 1-17. doi: 10.1007/s00426-003-0132-y

Jacoby, L. L., Lindsay, D. S., and Hessels, S. (2003). Item-specific control of automatic processes: stroop process dissociations. Psychon. Bull. Rev. 10, 634-644. doi: 10.3758/BF03196526

Jiménez, L., and Méndez, A. (2014). Even with time, conflict adaptation is not made of expectancies. Front. Psychol. 5:1042. doi: 10.3389/fpsyg.2014.01042

Levin, Y., and Tzelgov, J. (2014). Conflict components of the stroop effect and their “control.” Front. Psychol. 5:463. doi: 10.3389/fpsyg.2014.00463

Mayr, U., Awh, E., and Laurey, P. (2003). Conflict adaptation effects in the absence of executive control. Nat. Neurosci. 6, 450-452. doi: 10.1038/nn1051

Mordkoff, J. T. (2012). Observation: three reasons to avoid having half of the trials be congruent in a four-alternative forced-choice experiment on sequential modulation. Psychon. Bull. Rev. 19, 750-757. doi: 10.3758/s13423-0120257-3

Schmidt, J. R. (2013a). Questioning conflict adaptation: proportion congruent and Gratton effects reconsidered. Psychon. Bull. Rev. 20, 615-630. doi: 10.3758/s13423-012-0373-0

Schmidt, J. R. (2013b). The Parallel Episodic Processing (PEP) model: dissociating contingency and conflict adaptation in the item-specific proportion congruent paradigm. Acta Psychol. 142, 119-126. doi: 10.1016/j.actpsy.2012.11.004

Schmidt, J. R. (2014a). Contingencies and attentional capture: the importance of matching stimulus informativeness in the item-specific proportion congruent task. Front. Psychol. 5:540. doi: 10.3389/fpsyg.2014.00540

Schmidt, J. R. (2014b). Contingency and congruency switch in the congruency sequence effect: a reply to Blais, Stefanidi, and Brewer (2014). Front. Psychol. 5:1405. doi: 10.3389/fpsyg.2014.01405

Schmidt, J. R., and Besner, D. (2008). The Stroop effect: why proportion congruent has nothing to do with congruency and everything to do with contingency. J. Exp. Psychol. 34, 514-523. doi: 10.1037/0278-7393.34.3.514

Schmidt, J. R., and De Houwer, J. (2011). Now you see it, now you don't: controlling for contingencies and stimulus repetitions eliminates the Gratton effect. Acta Psychol. 138, 176-186. doi: 10.1016/j.actpsy.2011.06.002

Schmidt, J. R., Lemercier, C., and De Houwer, J. (2014). Context-specific temporal learning with non-conflict stimuli: proof-of-principle for a learning account of context-specific proportion congruent effects. Front. Psychol. 5:1241. doi: 10.3389/fpsyg.2014.01241

Spapé, M. M., and Hommel, B. (2014). Sequential modulations of the Simon effect depend on episodic retrieval. Front. Psychol. 5:855. doi: 10.3389/fpsyg.2014.00855

Van Lierde, E., Desender, K., and Van den Bussche, E. (2014). Is conflict adaptation triggered by feature repetitions? an unexpected finding. Front. Psychol. 5:1358. doi: 10.3389/fpsyg.2014.01358

Wendt, M., Luna-Rodriguez, A., and Jacobsen, T. (2014). Sequential modulation of distractor-interference produced by semantic generalization of stimulus features. Front. Psychol. 5:1271. doi: 10.3389/fpsyg.2014.01271

Conflict of Interest Statement: The authors declare that the research was conducted in the absence of any commercial or financial relationships that could be construed as a potential conflict of interest.

Received: 02 February 2015; accepted: 03 February 2015; published online: 18 February 2015.

Citation: Schmidt JR, Notebaert W and Van Den Bussche E (2015) Is conflict adaptation an illusion? Front. Psychol. 6:172. doi: 10.3389/fpsyg.2015.00172

This article was submitted to Cognition, a section of the journal Frontiers in Psychology.

Copyright (c) 2015 Schmidt, Notebaert and Van Den Bussche. This is an openaccess article distributed under the terms of the Creative Commons Attribution License (CC BY). The use, distribution or reproduction in other forums is permitted, provided the original author(s) or licensor are credited and that the original publication in this journal is cited, in accordance with accepted academic practice. No use, distribution or reproduction is permitted which does not comply with these terms. 